\title{
Centrosomal-Ciliary Gene CEP290/NPHP6 Mutations Result in Blindness With Unexpected Sparing of Photoreceptors and Visual Brain: Implications for Therapy of Leber Congenital Amaurosis
}

\author{
Artur V. Cideciyan, ${ }^{1 *}$ Tomas S. Aleman, ${ }^{1}$ Samuel G. Jacobson, ${ }^{1}$ Hemant Khanna, ${ }^{2}$ Alexander Sumaroka, ${ }^{1}$ \\ Geoffrey K. Aguirre, ${ }^{3}$ Sharon B. Schwartz, ${ }^{1}$ Elizabeth A.M. Windsor, ${ }^{1}$ Shirley $\mathrm{He},{ }^{2}$ Bo Chang, ${ }^{4}$ \\ Edwin M. Stone, ${ }^{5}$ and Anand Swaroop ${ }^{2}$ \\ ${ }^{1}$ Scheie Eye Institute, Department of Ophthalmology, University of Pennsylvania, Philadelphia, Pennsylvania; ${ }^{2}$ Department of Ophthalmology \\ and Visual Sciences and Human Genetics, W.K. Kellogg Eye Center, University of Michigan, Ann Arbor, Michigan; ${ }^{3}$ Department of Neurology, \\ University of Pennsylvania, Philadelphia, Pennsylvania; ${ }^{4}$ The Jackson Laboratory, Bar Harbor, Maine; ${ }^{5}$ Howard Hughes Medical Institute \\ and Department of Ophthalmology, University of Iowa Carver College of Medicine, Iowa City, Iowa
}

Communicated by Andrew Wilkie

Mutations in the centrosomal-ciliary gene CEP290/NPHP6 are associated with Joubert syndrome and are the most common cause of the childhood recessive blindness known as Leber congenital amaurosis (LCA). An in-frame deletion in Cep290 shows rapid degeneration in the rod-rich mouse retina. To explore the mechanisms of the human retinal disease, we studied CEP290-LCA in patients of different ages (7-48 years) and compared results to Cep290-mutant mice. Unexpectedly, blind CEP290-mutant human retinas retained photoreceptor and inner laminar architecture in the cone-rich central retina, independent of severity of visual loss. Surrounding the cone-rich island was photoreceptor loss and distorted retina, suggesting neural-glial remodeling. The mutant mouse retina at 4-6 weeks of age showed similar features of retinal remodeling, with altered neural and synaptic laminae and Muller glial activation. The visual brain pathways in CEP290-LCA were anatomically intact. Our findings of preserved foveal cones and visual brain anatomy in LCA with CEP290 mutations, despite severe blindness and rapid rod cell death, suggest an opportunity for visual restoration of central vision in this common form of inherited blindness. Hum Mutat 28(11), 1074-1083, 2007. Published 2007 Wiley-Liss, Inc. ${ }^{\dagger}$

KEY WORDS: CEP290; cilium; photoreceptor; neural remodeling; NPHP6; blindness; retina; Leber congenital amaurosis

\section{INTRODUCTION}

CEP290/NPHP6 (MIM\# 610142) mutations are a newlyidentified cause of Joubert syndrome (JSRD), a childhood-onset autosomal recessive disease with renal, cerebellar, and retinal manifestations [Sayer et al., 2006; Valente et al., 2006]. CEP290 exhibits centrosomal and ciliary localization and is considered among a series of genes involved in cystic kidney disease, developmental malformation of the brain, and retinal degenerations [Badano et al., 2005; Eley et al., 2005; Hildebrandt and Otto, 2005; Pan et al., 2005; Saunier et al., 2005; Bisgrove and Yost, 2006; Parisi et al., 2007]. Coincident with the JSRD discovery was the finding of a Cep290 mutation in rd16, a spontaneous mouse mutant with early-onset retinal degeneration [Chang et al., 2006]. Then, CEP290 mutations were identified as the most common cause of Leber congenital amaurosis (LCA), accounting for at least $21 \%$ of this nonsyndromic congenital retinal blindness [Cremers et al., 2002; den Hollander et al., 2006; Perrault et al., 2007].
Currently, little is known about the disease expression in the CEP290 form of LCA (or the retinopathy of JSRD) other than it causes severe human blindness. In contrast, details of the phenotype of three other molecular forms, RPE65-LCA (MIM\#

Received 8 February 2007; accepted revised manuscript 1 May 2007.

*Correspondence to: Artur V. Cideciyan, Scheie Eye Institute, 51 North 39th Street, Philadelphia, PA 19104.

E-mail: cideciya@mail.med.upenn.edu

Grant sponsors: National Institutes of Health; Macula Vision Research Foundation; Foundation Fighting Blindness, Inc.; Macular Disease Foundation; Hope for Vision; Howard Hughes Medical Institute; Research to Prevent Blindness; Chatlos Foundation; George M. O'Brien Kidney Research Center; Ruth and Milton Steinbach Award, Alcon Research Institute; Grousbeck Family Foundation.

DOI 10.1002/humu.20565

Published online 6 June 2007 in Wiley InterScience (www.interscience. wiley.com).

TThis article is a US Government work and, as such, is in the public domain in the United States of America. 
180069), CRB1-LCA (MIM\# 604210), and RDH12-LCA (MIM\# 608830), have been explored [Jacobson et al., 2003, 2005, 2007a]. RPE65-LCA shows structurally intact retina and is a candidate for human somatic retinal gene therapy [Jacobson et al., 2005], whereas CRB1-LCA and RDH12-LCA show disorganized retina lacking normal lamination and may be less approachable by such therapeutic strategies [Jacobson et al., 2003, 2007a]. The availability of the $r d 16$ mouse model for proof-of-concept studies and a major proportion of LCA being caused by CEP290 mutations warrant asking questions about the feasibility of therapy in this human disease.

\section{MATERIALS AND METHODS Human Studies-Mutation Screening}

Patients with the clinical diagnosis of LCA and CEP290 mutations (GenBank accession number NM_025114.3), an LCA patient with RPGRIP1 mutation (GenBank accession number NM_020366.3), and retinitis pigmentosa (RP) patients with RPGR mutations (GenBank accession number AF286472.1) or of unknown genotype were included (Table 1). Blood samples were obtained from patients and DNA was prepared from peripheral blood leukocytes by previously described procedures [Lotery et al., 2001]. All CEP290 probands were screened and found to be negative for disease-causing mutations in other LCA genes including AIPL1, CRB1, CRX, GUCY2D, RDH12, RPE65, and RPGRIP1 using reported methods [Lotery et al., 2001]. RPGR mutation screening has been described [Breuer et al., 2002]. Informed consent was given by all subjects; institutional approval was obtained, and the tenets of the Declaration of Helsinki were followed.

\section{Cross-Sectional Imaging With Optical Coherence Tomography}

In vivo microstructure of the retina was quantified with optical coherence tomography (OCT) (OCT3; Zeiss Humphrey Instruments, Dublin, CA) with a depth resolution of $\sim 8 \mu \mathrm{m}$. The principles of the method and our recording and analysis techniques have been published [Huang et al., 1998; Jacobson et al., 2003, 2005]. In all subjects, overlapping OCT scans of $4.5 \mathrm{~mm}$ in length were used to cover horizontal and vertical meridians up to $9 \mathrm{~mm}$ eccentricity from the fovea; in this context, eccentricity is defined as the radial distance of a retinal location from the anatomical center of the fovea. In a subset of patients, dense raster scans were performed to sample a $18 \times 12 \mathrm{~mm}^{2}$ region of the retina centered on the fovea. At least three OCT scans were obtained at each retinal location. At extrafoveal retinal regions, two or three repeated scans were averaged to increase signal-to-noise ratio and allow better definition of retinal laminae. At the fovea, only scans

TABLE 1. Clinical and Molecular Characteristics of the Patients*

\begin{tabular}{|c|c|c|c|c|c|c|c|c|c|}
\hline \multirow{2}{*}{$\begin{array}{l}\text { Gene } \\
\text { Patient }\end{array}$} & \multirow{2}{*}{$\begin{array}{c}\text { Age } \\
\text { (years)/gender }\end{array}$} & \multirow[b]{2}{*}{ Nucleotide change } & \multirow[b]{2}{*}{ Protein change } & \multicolumn{2}{|c|}{ Visual acuity $^{a}$} & \multicolumn{2}{|c|}{ Refraction $^{b}$} & \multicolumn{2}{|c|}{$\begin{array}{c}\text { Kinetic visual } \\
\text { field extent }(\mathrm{V}-4 e)^{c}\end{array}$} \\
\hline & & & & RE & LE & RE & LE & RE & LE \\
\hline \multicolumn{10}{|l|}{$C E P 290^{\mathrm{d}}$} \\
\hline P1 & 7/M & c. $2991+1655 A>G$ & Splice defect ${ }^{e}$ & NLP & NLP & +7.00 & +7.75 & ND & ND \\
\hline P2 & $11 / F$ & $\begin{array}{l}\text { c. } 2991+1655 A>G \\
\text { c. } 2991+1655 A>G\end{array}$ & $\begin{array}{l}\text { Splice defect } \\
\text { Splice defect }\end{array}$ & HM & HM & +5.25 & +6.00 & ND & ND \\
\hline P3 & $14 / M$ & $\begin{array}{l}\text { c. } 2991+1655 A>G \\
\text { c. } 4882 \mathrm{C}>\mathrm{T}\end{array}$ & $\begin{array}{l}\text { Splice defect } \\
\text { p.Q1628X }\end{array}$ & NLP & NLP & +5.50 & +5.50 & ND & ND \\
\hline$P 4^{f}$ & $17 / \mathrm{M}$ & $\begin{array}{l}\text { c. } 2991+1655 A>G \\
\text { c. } 5668 G>T\end{array}$ & $\begin{array}{l}\text { Splice defect } \\
\text { p.G1890X }\end{array}$ & $20 / 1200$ & $20 / 800$ & +6.50 & $\mathbf{N} / \mathbf{A}$ & ND & ND \\
\hline$P 5^{f}$ & $19 / F$ & $\begin{array}{l}\text { c. } 2991+1655 A>G \\
\text { c. } 5668 G>T\end{array}$ & $\begin{array}{l}\text { Splice defect } \\
\text { p.G1890X }\end{array}$ & $20 / 63$ & $20 / 50$ & +2.75 & +3.25 & 13 & 15 \\
\hline P6 & $20 / F$ & $\begin{array}{l}\text { c. } 2991+1655 A>G \\
\text { c. } 2991+1655 A>G\end{array}$ & $\begin{array}{l}\text { Splice defect } \\
\text { Splice defect }\end{array}$ & $20 / 400$ & $20 / 50$ & +7.75 & +4.00 & 8 & 8 \\
\hline P7 & $26 / M$ & $\begin{array}{l}\text { c. } 2991+1655 A>G \\
\text { c.1260_1264deITAAAG }\end{array}$ & $\begin{array}{l}\text { Splice defect } \\
\text { p.T420fs }\end{array}$ & LP & LP & +4.00 & +4.00 & 2 & 4 \\
\hline & $48 / \mathrm{M}$ & c. $2991+1655 \mathrm{~A}>\mathrm{G}$ & Splice defect ${ }^{e}$ & NLP & NLP & +6.00 & +6.00 & ND & ND \\
\hline \multicolumn{10}{|l|}{ RPGRIP1 ${ }^{\mathrm{g}}$} \\
\hline P9 ${ }^{h}$ & $21 / \mathrm{M}$ & $\begin{array}{l}\text { c. } 3632 \mathrm{~T}>\mathrm{A} \\
\text { c.3632T }>\mathrm{A}\end{array}$ & $\begin{array}{l}\text { p.V1211E } \\
\text { p.V1211E }\end{array}$ & $20 / 80$ & $20 / 100$ & +2.75 & +3.50 & $<1$ & $<\mathbf{1}$ \\
\hline \multicolumn{10}{|l|}{$R P G R^{i}$} \\
\hline P10 & $10 / M$ & $\begin{array}{l}\text { c.2236_2237delGA } \\
\text { (g.ORF15+483_484delGA) }\end{array}$ & $\begin{array}{c}\text { p.E746fs } \\
\text { (p.ORF15+ } \\
\text { E161fs) }\end{array}$ & $20 / 40$ & $20 / 40$ & -2.00 & -1.75 & 85 & 90 \\
\hline P11 & $48 / M$ & $\begin{array}{l}\text { c. } 2260 \mathrm{G}>\mathrm{T} \\
(\mathrm{g} . \mathrm{ORF} 15+507 \mathrm{G}>\mathrm{T})\end{array}$ & $\begin{array}{c}\text { p.E754X } \\
\text { (p.ORF15+ } \\
\text { E169X) }\end{array}$ & HM & HM & -1.00 & -1.25 & 18 & 13 \\
\hline
\end{tabular}

* Second allele is not yet determined in Patients P1 and P8. Novel mutation is indicated in bold.

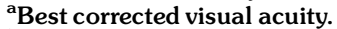

b Spherical equivalent.

'Expressed as a percentage of normal mean of V-4e target; 2 SD below normal equals $90 \%$.

${ }^{\mathrm{d}}$ Nucleotide positions are based on GenBank sequence NM_025114.3, with the A of the start codon (ATG) designated as +1 .

${ }^{e}$ Creates a new splice site in intron 26 that causes a premature stop at position 998.

${ }^{\text {f}}$ Patients are siblings.

gNucleotide position is based on GenBank sequence NM_020366.3, with the A of the start codon (ATG) designated as +1.

${ }^{\mathrm{h}}$ Some patient information was provided previously: for Patient P9 [Jacobson et al., 2007 b].

${ }^{\mathrm{i}}$ Nucleotide positions are on GenBank sequence BK005711.1, with the A of the start codon (ATG) designated as +1 . The traditional nomenclature, shown in parentheses, is based on accession numbers U57629.1 and AF286472.1.

$\mathrm{RE}$, right eye; LE, left eye; ND, not detectable; N/A, not available; HM, hand motions; LP, light perception; NLP, no light perception. 
showing the deepest pit were used. A video fundus image was acquired and saved with each OCT scan by the commercial software. In addition, the fundus video visible during the complete session was recorded continuously on a video cassette recorder.

Thickness of nuclear layers was defined as previously published [Huang et al., 1998; Jacobson et al., 2003, 2005]. The presence/ absence of cilium signal was determined. In normal subjects, the signal corresponding to the retinal pigment epithelium (RPE) was assumed to be the last peak within the two- or three-peaked scattering signal complex [Huang et al., 1998], deep in the retina. In abnormal retinas, the presumed RPE peak was sometimes the only signal peak deep in the retina; in other cases, it was apposed by other major peaks. In the latter case, RPE peak was specified manually by considering the properties of the backscattering signal originating from layers vitread and sclerad to it.

\section{En Face Imaging With Scanning Laser Ophthalmoscope}

Reflectance imaging with near-infrared (NIR) light and autofluorescence (AF) imaging with NIR was obtained with a second generation confocal scanning laser ophthalmoscope (SLO) (HRA2; Heidelberg Engineering, Dossenheim, Germany) as previously described [Cideciyan et al., 2007]. The "high-speed" mode of the instrument was used, with which a $30^{\circ} \times 30^{\circ}$ region of the retina is sampled onto a $768 \times 768$ pixel image and consecutive frames can be obtained at the rate of $9 \mathrm{~Hz}$. Images were exported from the manufacturer's software and analyzed as described [Cideciyan et al., 2007]. In a subset of six CEP290-LCA patients with quantifiable NIR-AF intensities, the strength of the parafoveal boundary was estimated with the discrete derivative of the intensity profile along the horizontal meridian performed after smoothing images with a 25-pixel (1 degree) radius Gaussian filter. The patient results were compared to normals $(n=15)$.

\section{Visual Perception With Psychophysics}

Visual perception, when measurable, was determined with psychophysical methods. Specifically, two-color dark-adapted perimetry $(500-\mathrm{nm}$ and $650-\mathrm{nm}$ stimuli) was used to assay the sensitivity of rod photoreceptor-mediated (night) vision, and 600 . $\mathrm{nm}$ stimuli in the light-adapted state to measure cone photoreceptor-mediated (day) vision. Details of the visual function techniques and analysis methods have been published [Jacobson et al., 1986].

\section{Brain Anatomy With MRI}

A 3.0 Tesla Siemens Trio (Siemens, Malvern, PA), and an eightchannel head coil were used for MRI acquisition. Intraorbital optic nerve diameter was assessed by direct measurement of the interpial diameter of the optic nerve on high-resolution $(0.375$ $\times 0.375 \times 2.2 \mathrm{~mm}) \mathrm{T} 2$-weighted anatomical images. Voxel-based morphometry [Ashburner and Friston, 2000] was performed upon the log Jacobian measure obtained after diffeomorphic warping [Avants and Gee, 2004] of the T1-weighted MPRAGE images from patients and a population of normal subjects to a representative brain image. The average deformation score was also obtained for normal subjects and patients from within a region of interest defined within the occipital lobe white matter.

\section{Animal Studies-Histology and Immunohistochemistry}

Retinal tissue from wild-type (WT) and $r d 16$ mice (ages 19 days to 14 months) and retinal sections from a cynomolgus monkey (age 2 years) were used. For light microscopy, mouse eyes were placed in Bouin's fixative overnight, embedded in paraffin, and sectioned; sections were stained with hematoxylin and eosin [Chang et al., 2006]. Central (within $1 \mathrm{~mm}$ of the optic nerve) and midretinal (1-3 mm from the optic nerve) regions were examined. Antibodies used in localization studies were as follow: CEP290 antibody, which has been described [Chang et al., 2006]; glial fibrillary acidic protein (GFAP) antibody obtained from Sigma (St. Louis, MO); and Rhodamine-labeled peanut agglutinin (PNA) from Vector Laboratories (Burlingame, CA). Nuclei were labeled using 4',6' -diamino-2-phenylindole (DAPI). For immunohistochemistry of the monkey retina, paraffin-fixed sections were deparaffinized and rehydrated as follows: $20 \mathrm{~min}$ in xylene; $10 \mathrm{~min}$ in $100 \%$ ethanol; $3 \mathrm{~min}$ in $95 \%$ ethanol; $3 \mathrm{~min}$ in $80 \%$ ethanol, and $3 \mathrm{~min}$ in $70 \%$ ethanol. After rinsing in PBS, the slides were boiled in $10 \mathrm{mM} \mathrm{Na}$ citrate $/ 0.1 \%$ Tween-20 in a container with boiling water for $20 \mathrm{~min}$. Sections were blocked with 5\% goat serum, and incubated in succession with primary antibodies (overnight) and appropriate fluorochrome-labeled secondary antibodies (Invitrogen, Carlsbad, CA).

\section{RESULTS \\ Ciliopathies and Retinal Degeneration: Comparison With CEP290-Mutant Retina}

Increasing numbers of genetic retinal degenerations are being recognized to affect the specialized photoreceptor cilium with or without disease effects on primary or motile cilia [Badano et al., 2005; Bisgrove and Yost, 2006]. We asked how the microstructure of the human CEP290-mutant central retina compared with two other ciliopathies associated with retinal degeneration-RPGR mutation, causing X-linked RP; and RPGRIP1 mutation, causing a rare form of LCA [Cremers et al., 2002; Wright and Shu, in press]. Notably, both RPGR and RPGRIP1 associate with CEP290 in mammalian retina [Chang et al., 2006]. The normal human central retina in cross-section shows a depression or pit at the cone photoreceptor-rich fovea and adjacent laminar architecture representing nuclei, synapses, and nerve axons (Fig. 1A). Highlighted are the photoreceptor outer nuclear layer (ONL, blue) and the deeper optical signal attributable to the cilium near the junction of photoreceptor inner and outer segments (IS/OS) (yellow). The normal complement of rod (gray) and cone (red) vision is depicted above the retinal cross-sectional image. Two RPGR-mutant retinas represent different disease stages (Fig. 1B and C). An early stage in Patient P10, a 10-year-old boy, shows impaired rod but nearly-normal cone vision; photoreceptor lamination approximates normal at the fovea but declines with distance therefrom (Fig. 1B). A later stage RPGR-mutant retina in Patient P11, a 48-year-old man, shows no measurable rod or cone vision and, anatomically, there is a deep foveal pit due to photoreceptor losses at this locus and across the central retina (Fig. 1C).

The RPGRIP1-mutant retina from Patient P9, a 19-year-old man with LCA [Jacobson et al., 2007b], has severely abnormal rod vision but some preserved cone vision limited to the foveal region (Fig. 1D). The cone photoreceptor layer at the fovea is within normal limits but declines with distance from the fovea. A signal from the cilium is detectable only near the foveal center. Two CEP290-LCA retinas also show retained central retinal structure but severely reduced vision. Patient P6, a 20-year-old, has no rod vision but some measurable cone vision. The cone photoreceptor layer in the central retina appears normal and there is a foveal cilium optical signal (Fig. 1E). Patient P1, a 7-year-old child with no perception of light, has retained the central photoreceptor nuclear layer but no discernible cilium signal (Fig. 1F), in contrast 


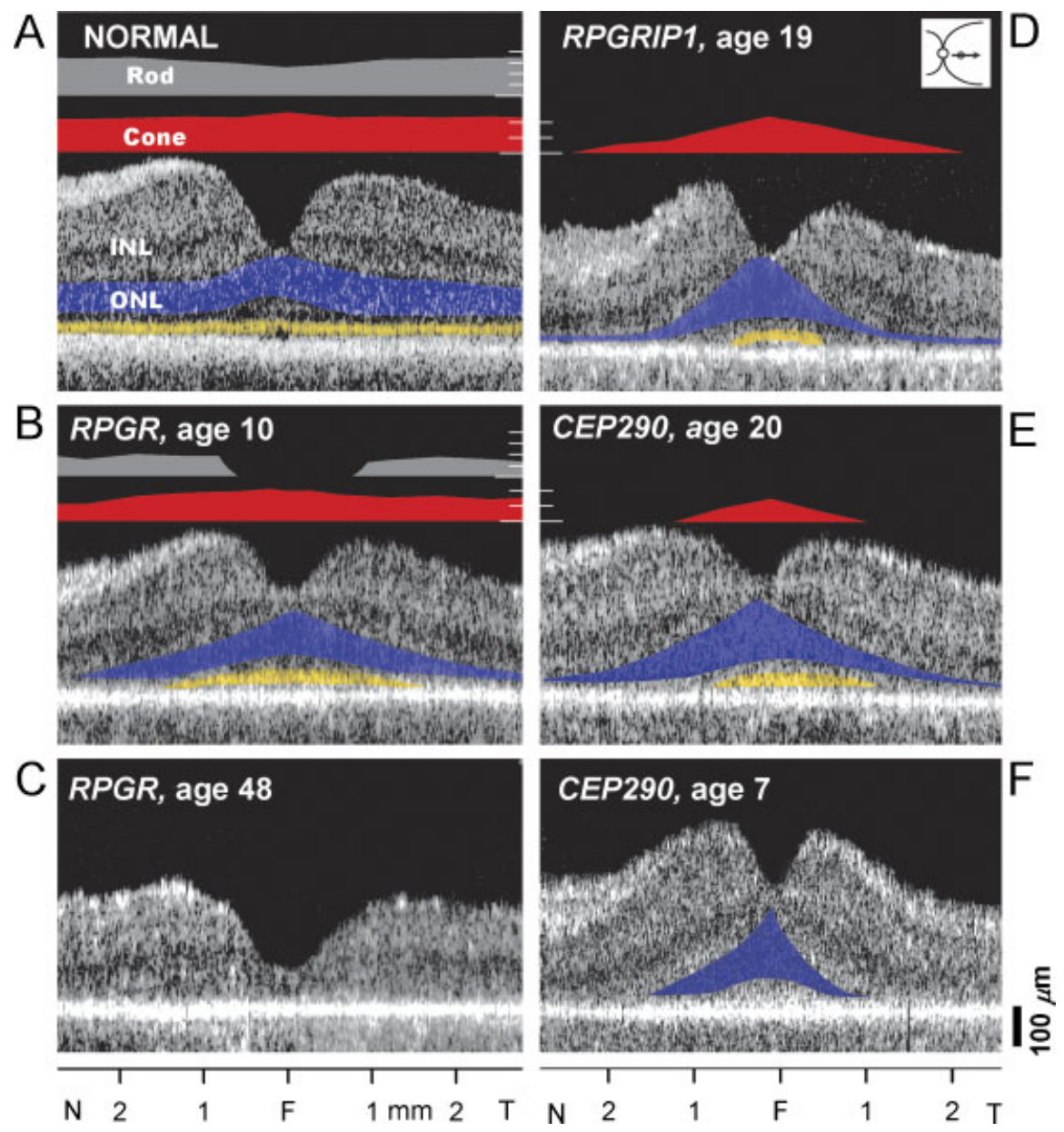

FIGURE 1. In vivo microscopy of the central retina and colocalized rod and cone vision along the horizontal meridian in a normal subject and individuals with different ciliopathies. A: Normal retina (age 20 years) with full complement of rod and cone function. B,C: RPGR-mutant retinas representing early (Patient P10) and late (Patient P11) disease stages. D: RPGRIP1-mutant retina (Patient P9) showing no rod function and preserved cone function only at the fovea. This is a previously published scan [Jacobson et al., 2007b] with new analyses. E: CEP290-mutant retina (Patient P6) showing no rod function and preserved cone function only at the fovea. F: CEP290-mutant retina (Patient P1) with no measurable rod or cone function. F, fovea; ONL, outer nuclear layer; N, nasal retina; INL, inner nuclear layer; T, temporal retina. ONL highlighted in blue. Cilium signal highlighted in yellow. Sensitivities for rods (gray bar; dark-adapted, $500-\mathrm{nm}$ stimuli; range displayed is from 1.5 to $5.5 \log$ units; white tics correspond to 1 log unit increments), and cones (red bar; light-adapted, 600 -nm stimuli; range from 0 to 2.8 log units; white tics correspond to 1 log unit increments) are shown above the central retinal scans. Inset, D: Location of the scans and visual function on a schematic of the left retina showing vascular arcades, optic nerve, and fovea.

with the late-stage RPGR-mutant retina, with loss of retinal tissue accompanying loss of vision (Fig. 1C).

\section{Spared Retina and RPE at the Cone Photoreceptor-Rich Foveal Region}

Topography of the photoreceptor layer in normal human retina has a central peak and declines with distance from the fovea; a shallower rate of decline in the superior direction results in anisotropic contour lines extending superiorly (Fig. 2A, inset). The CEP290-mutant retina of Patient P5, a 19-year-old, retains only a horizontally-elongated central island of measurable ONL; there is a normal peak and a slightly greater extent temporally than nasally. ONL thickness was analyzed across the horizontal meridian in normal subjects ( $n=16$; ages $6-58$ years) and the CEP290-mutant retinas (Fig. 2B). At the fovea, six CEP290mutant retinas showed normal ONL thickness whereas two (Patients P2 and P4) had slightly greater than normal peak thickness. ONL thickness in all CEP290-mutant retinas steeply declined and became less than normal between 0.5 to $1 \mathrm{~mm}$ eccentricity. Five of the eight CEP290-mutant retinas had detectable signal from the cilium; the three without this optical signal also had no light perception. Patient P8, the 48-year-old, had the most limited extent of ONL (Fig. 2B).

The topography of RPE integrity in CEP290-mutant eyes was defined with NIR-AF imaging; the signal is believed to originate from melanin and melanolipofuscin granules in the RPE and melanin in the choroid [Keilhauer and Delori, 2006; Cideciyan et al., 2007]. In normal human eyes, NIR-AF images show a circular central region of homogeneous appearance and higher brightness extending from the fovea. With increasing eccentricity there is a decrease in brightness and an increase in appearance of choroidal features corresponding to the vasculature deep to the RPE (Fig. 2C, inset). NIR-AF topographies of CEP290-mutant eyes also showed a central region of higher intensity surrounded by a more peripheral region of lower intensity; however, there were 

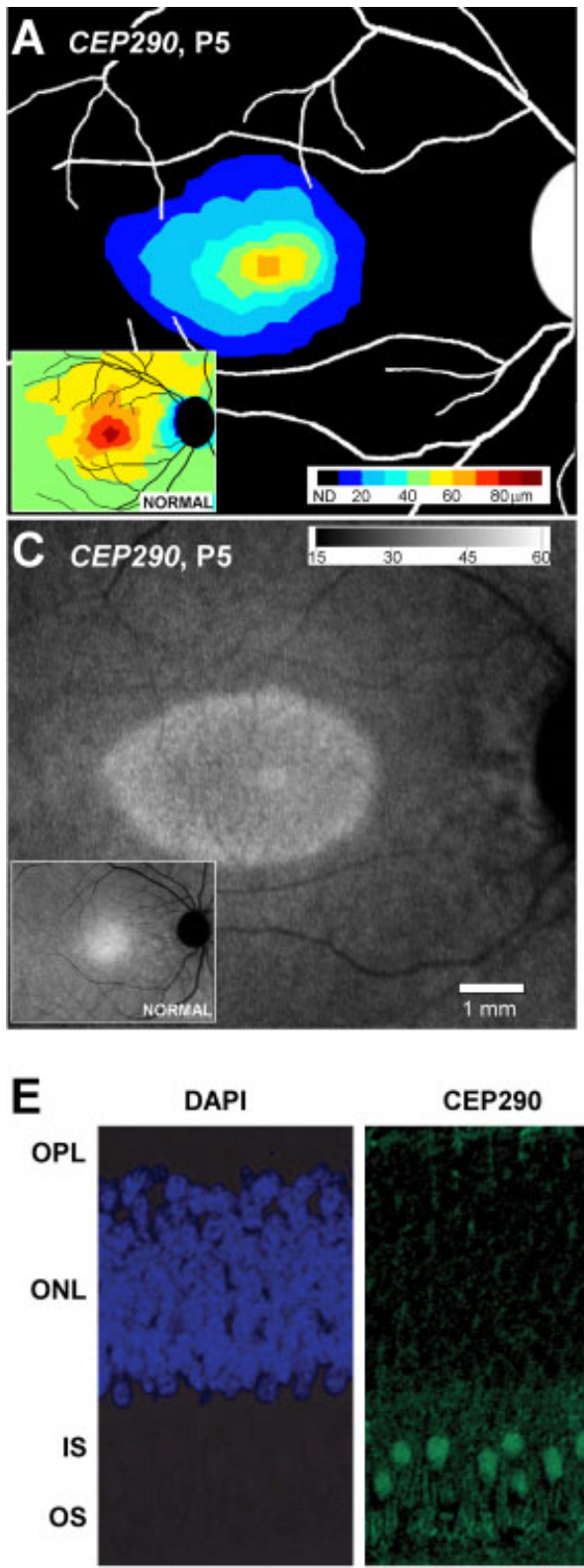

CEP290

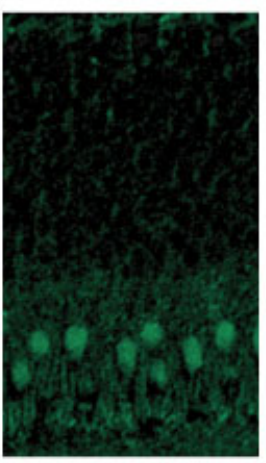

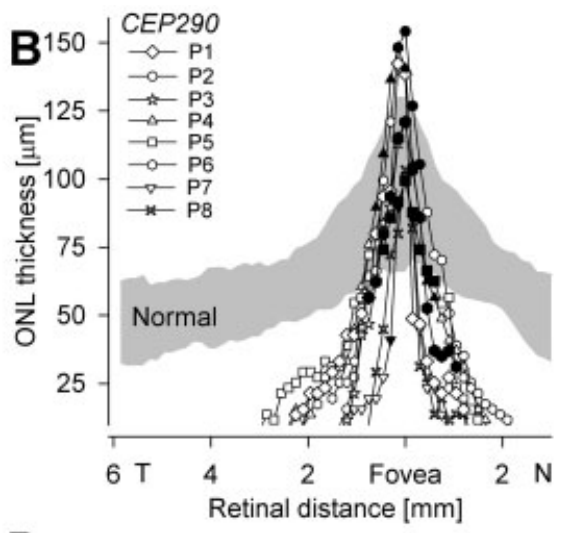

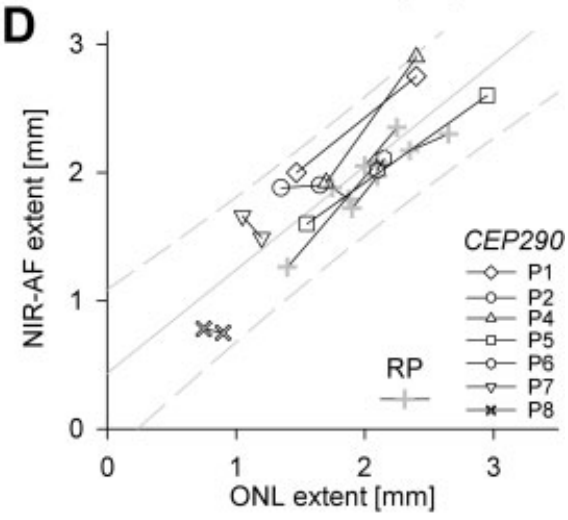

PNA

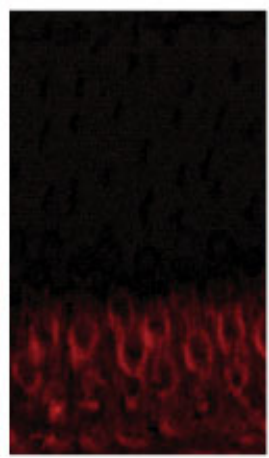

Merge

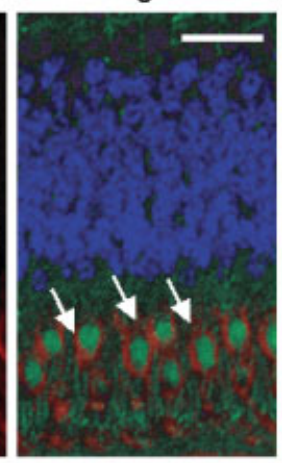

FIGURE 2. Spatial distribution of the extent of retained retinal photoreceptors and healthy RPE. A: Topography of ONL thickness in the CEP290-mutant retina of Patient P5 displayed in pseudocolor. The location of the optic nerve head and major retinal blood vessels are overlaid (white) for reference. Inset: Normal ONL thickness topography of a 28-year-old. B: ONL thickness profiles along the horizontal meridian in CEP290-mutant retinas; filled symbols correspond to retinal locations with a detectable cilium signal. Normal ONL thickness (mean \pm 2 SD) is depicted (gray). C: NIR-AF image of Patient P5 shown at the same spatial scale as in (A). Inset: NIRAF image of a representative normal subject (age 22 years). D: Relation of retained NIR-AF signal boundary and retained ONL thickness boundary in seven CEP290-mutant retinas and in five RP patients with a central island of function remaining. Two measurements made along the horizontal meridian toward the nasal and temporal directions from the fovea in each subject are connected by lines. Regression line (slope $=0.81, \mathrm{r}^{2}=0.76$ ) and $95 \%$ prediction intervals (dashed line) are estimated from all of the data shown. E: Immunofluorescence staining of CEP290 in cones of monkey retina. Sections were costained with CEP290 (green) and cone-specific marker, PNA (red). Immunodetection indicated colocalization of CEP290 with PNA (Merge; arrows) in cones. DAPI (blue) was used to stain the nuclei. Scale: $10 \mu \mathrm{m}$.

differences from normal (Fig. 2C). CEP290-mutant eyes had an elliptical region with a boundary that was qualitatively and quantitatively more abrupt (boundary strength $=5.4 \pm 1.2$ gray levels per degree) compared to that apparent normally in the parafoveal region $(3.1 \pm 1.4$ gray levels per degree). The spatial extent of the NIR-AF boundary along the horizontal meridian was compared to the extent of ONL thickness in CEP290-mutant retinas; there was a linear relationship (Fig. 2D) supporting the hypothesis of retained central retinal and RPE structural integrity in the presence of severely impaired visual function. For comparison, five patients with RP (of unknown genotype) who retained central islands of visual function were studied and results showed a similar abrupt boundary and a relationship between the extent of ONL thickness and NIR-AF boundary (Fig. 2D). 
The spared central retinal island of cone-rich human retina led to the question of whether there was indeed CEP290 immunolocalization in primate cones. As the reported CEP290 expression data (in rod-dominated mouse retina) did not clarify this [Chang et al., 2006; Sayer et al., 2006], we performed immunohistochemical analysis of monkey retina and found prominent immunostaining in cones, thereby confirming expression of CEP290 in both photoreceptor types (Fig. 2E). Cone staining of CEP290 was also detected in normal human retina (data not shown).

\section{Evidence for Retinal Remodeling in Human Peripheral CEP290-Mutant Retina}

Rod photoreceptor density normally increases while cone density declines with distance from the human fovea [Curcio et al., 1990]. At about $4-5 \mathrm{~mm}$ from the fovea, the ratio of rods to cones becomes more like that in mouse (25:1). In vivo microscopy in pericentral retina shows a relatively thick photoreceptor layer; the thinner inner nuclear layer (INL) is composed of bipolar, Muller glial, amacrine, and horizontal cell nuclei (Fig. 3A, inset left). CEP290-mutant retinas differed remarkably from normal (Fig. 3A, inset right). ONL was not detectable and INL appeared to thicken with increasing distance from the center. INL thickness in normal retina $(n=10$; ages $8-58$ years) increases over the first millimeter from the fovea and then thinning occurs at further eccentricities (Fig. 3A). All CEP290-mutant retinas had normal INL thickness in the first millimeter of eccentricity from the fovea. However, at greater eccentricity, six of eight showed abnormal thickening (rather than thinning) of the nuclear layer continuous with the INL.

Individual cross-sections of six CEP290-retinas at 5-7 mm temporal eccentricity illustrate the abnormal laminar pattern. The sections, ordered from left to right by total retinal thickness, show no obvious age dependence of this parameter (Fig. 3B). In contrast to normal lamination with thick ONL, thinner INL, and intervening synaptic laminae, CEP290-mutant retinas have an almost bilaminar reflectivity profile. There is a relatively thick hyporeflective nuclear layer (either an abnormal INL or combined INL-ONL with an unresolved plexiform layer between) and a more vitread layer of hyperreflectivity that is thicker than the normal inner plexiform layer (IPL). Neither of the layers showed a relationship of thickness or thinning to age; the oldest CEP290. mutant retina studied (Patient P8), however, had the thinnest nuclear layer. The deep multipeaked retinal signal we previously termed the outer-retina-choroid-complex [Huang et al., 1998, 2000; Jacobson et al., 2003, 2005] is reduced to a single-peaked component with generally higher backscatter than normal, suggesting loss of inner and outer segment signal and atrophy or demelanization of RPE.

\section{Inner Retinal Changes and Retinal Remodeling in rd16 Retina}

We asked whether the human laminar abnormalities in CEP290-mutant retina had any histological correlates in the rd16 mouse retina [Chang et al., 2006]. A 25-day-old $r d 16$ retina showed an expected reduction in ONL and photoreceptor outer segments as compared to 4-week-old WT retina. Based on the clues garnered from the human CEP290-LCA data, we reexamined the $r d 16$ mouse despite the earlier report that cellular layers other than the ONL were not dramatically changed [Chang et al., 2006]. We detected considerable thickening of the INL and IPL at a midretinal region (Fig. 3C). Interestingly, the nuclei of all cell types in the $r d 16$ retina appeared enlarged though the number

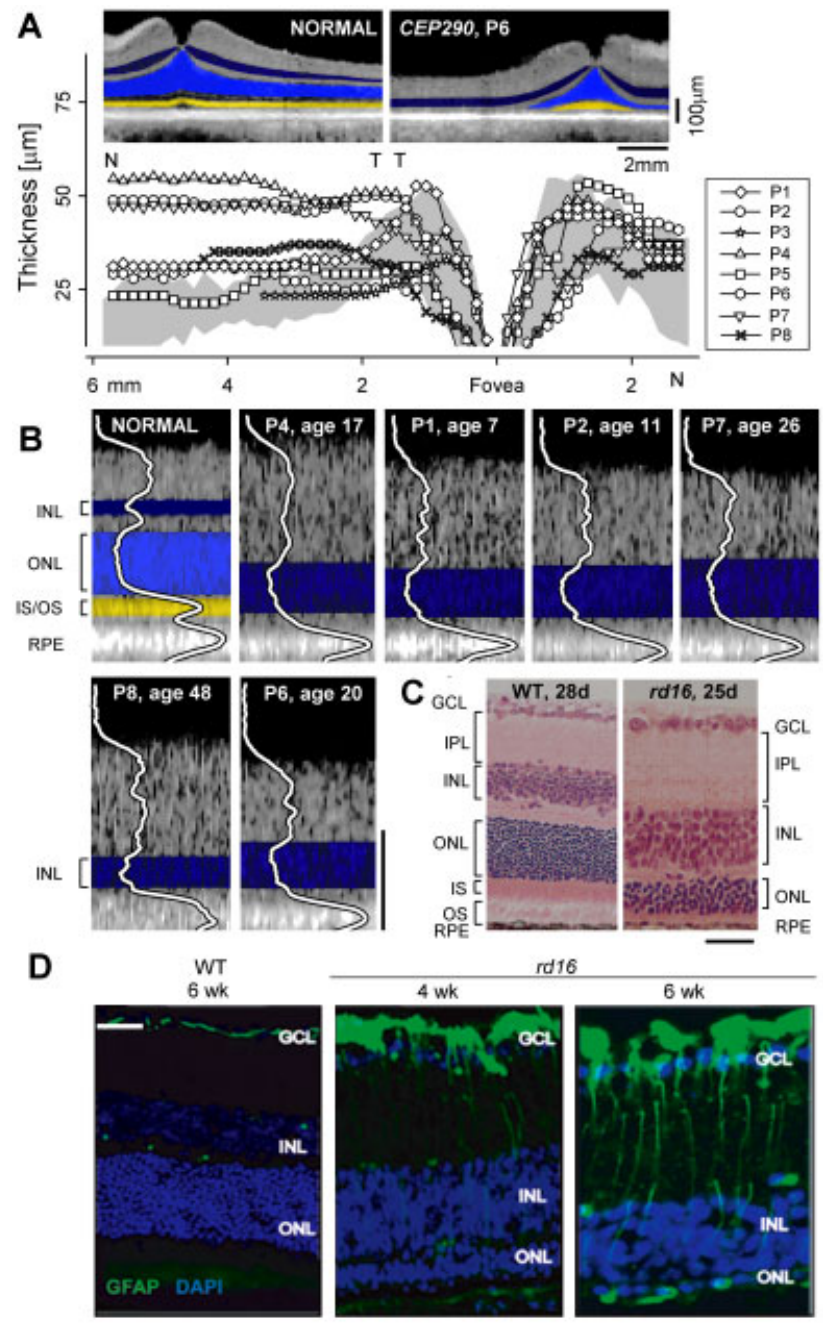

FIGURE 3. Human CEP290-mutant retina and murine rd16 retina show retinal remodeling. A: INL thickness profiles along the horizontal meridian in normal human retina $(n=8$; gray represents mean \pm 2 SD) and eight CEP290-mutant retinas (Patients $\mathrm{P1}-\mathrm{P} 8$, symbols). Inset above graph depicts an 11-mm-long cross-sectional image that shows the striking difference between lamination in a normal subject (age 26 years) and CEP290-LCA, Patient P6. B: Cross-sectional images from 5 to $7 \mathrm{~mm}$ in the temporal retina of a normal human retina (age 28 years) and six CEP290-mutant retinas. The abnormal retinas are ordered by total retinal thickness and show no age relationship. C: Retinal histology of a 4-week-old WT and a 25-day-old rd16 mouse retina at a midretinal region. Compared to WT, rd16 retina has a reduced ONL, IS, and OS. INL and IPL are thicker in $r d 16$ retina. Scale: $10 \mu \mathrm{m}$. D: Immunohistochemical analysis with fluorescent labeling using anti-GFAP antibody in WT and $r d 16$ retinas. GFAP labeling was restricted to astrocytes in the 6-week-old WT mouse, whereas activated glial reactivity (presumed due to remodeling of Muller cells in response to photoreceptor degeneration) was initiated by 4 weeks of age in rd16 and progressed with age. Nuclei are stained with DAPI (blue). Scale: $50 \mu \mathrm{m}$.

of rows of nuclei in INL and the ganglion cell layer (GCL) seemed unaltered. Examination of sections from $r d 16$ retinas from mice between 19 days and 14 months of age confirmed the findings at midretinal as well as central retinal regions (data not shown). To further characterize the changes in the inner retina of $r d 16$ mice, we looked at GFAP immunolabeling, a sensitive indicator of retinopathy detectable in activated Muller glial cells [Bringmann 
and Reichenbach, 2001]. In 6-week-old WT mouse retina, GFAP staining is primarily restricted to astrocytes; however, a notable increase in GFAP reactivity was observed in 4-week-old and 6-week-old $r d 16$ retinas (Fig. 3D).

\section{Visual Brain Pathway Anatomy Is Intact}

Retinal blindness at birth or shortly thereafter has been associated with atrophy of the optic nerves and chiasm as well as alterations in cortical gray and white matter [Noppeney et al.,
2005; Shimony et al., 2006]. We asked whether this occurs in CEP290-LCA. With MRI we studied Patients P4 (age 18 years) and P8 (age 48 years), who represent early and later retinal disease stages, respectively. Retinal structure in the two stages of CEP290. LCA disease is schematically depicted compared to normal (Fig. 4A). The visual pathway structures appeared normal in MRI images. The interpial optic nerve diameter in Patients P4 and P8 was normal (Fig. 4B), as defined by measurements from our normal subjects and published data [Karim et al., 2004]. This finding suggested there are normal numbers of ganglion cell axons that

\section{A RETINA}

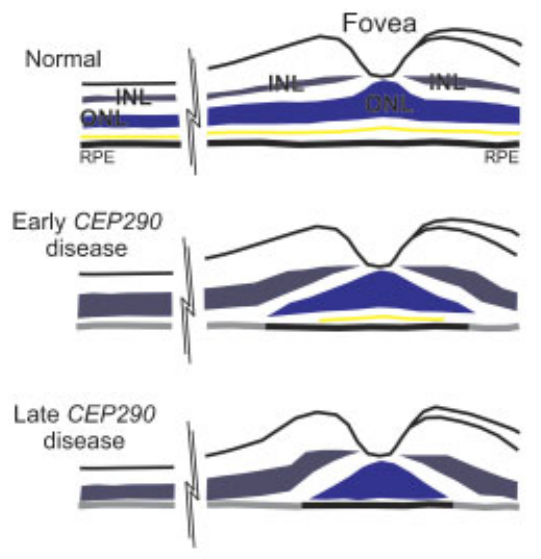

B OPTIC NERVE

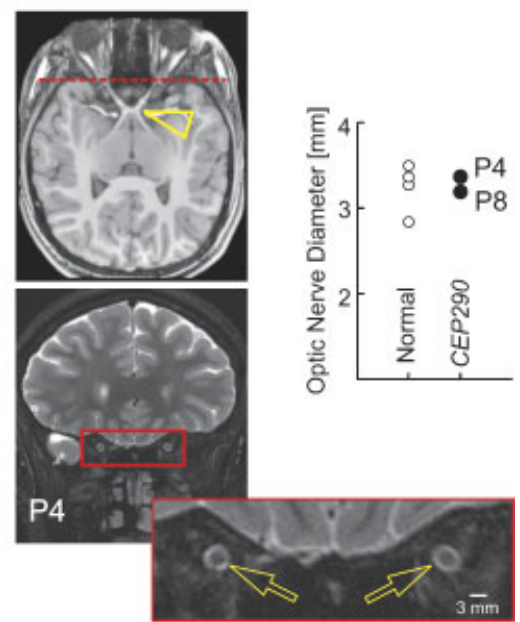

\section{OCCIPITAL WHITE MATTER} Registered Anatomy
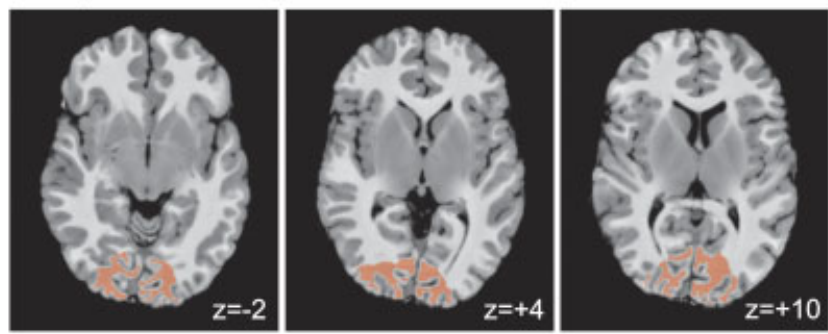

$\log$ Jacobian
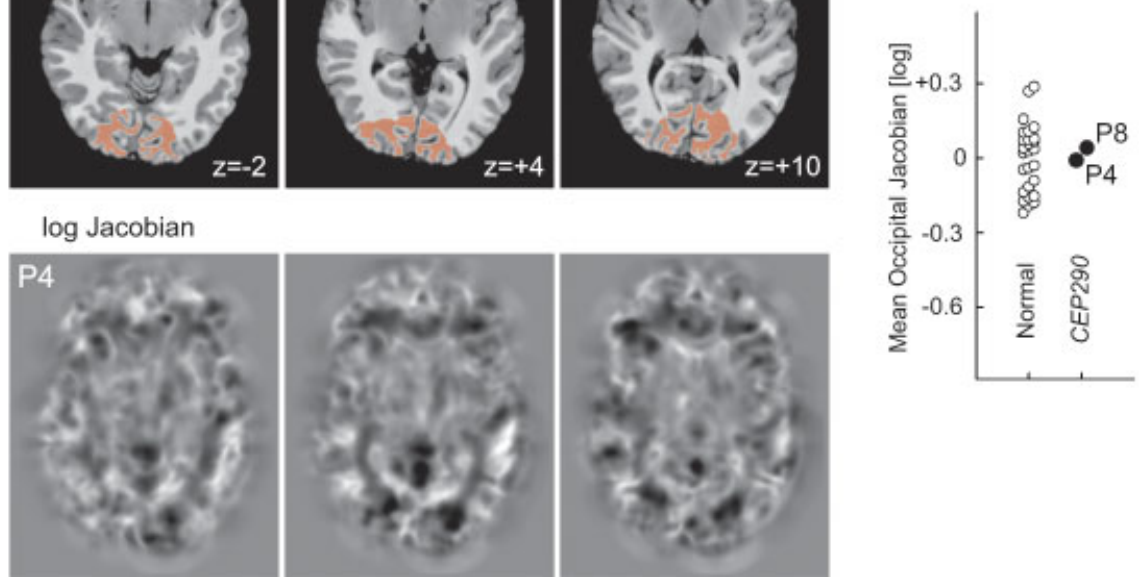

FIGURE 4. Visual pathway anatomy in human CEP290 disease from retina to cortex. A: Retina. Schematic representation of early and later CEP290-LCA retinal disease stages in central and more peripheral temporal (left) retina. Early stage: at and near the cone-rich fovea, there is a normal ONL (blue), detectable IS/OS (yellow), and a normally melanized RPE (black). Temporal retina lacks ONL and shows abnormal thickening of inner layers; RPE is demelanized (gray). Later stage: there is contraction of preserved retina and RPE and loss of IS/OS signal. Temporal retina has abnormal lamination but is thinner. B: Optic nerve anatomy. A normal-appearing optic chiasm (arrowhead) was observed on T1-imaging in Patient P4. High-resolution T2-weighted axial and coronal images were obtained through the optic nerves. The position of the coronal slice displayed is indicated by the dashed line on the axial image. The cross-sectional diameter of the interpial optic nerve (arrows) was estimated at three positions along each nerve, and the average diameter was within the range of normal. C: Whole-brain morphometric analysis. The T1-weighted anatomical images from CEP290-LCA and controls were warped to a representative template (top row). The (log) determinant of the Jacobian matrix calculated during warping for each subject (bottom row) indexes the degree to which cerebral tissue is smaller or larger than the template image. No significant deviation from control measures was seen in both CEP290-LCA (Patient P4 data illustrated). A focused analysis was conducted within occipital lobe white matter (red on the registered anatomy). The z-position ( $\mathrm{mm}$ ) of each axial slice relative to the anterior commissure is indicated. The average (log) Jacobian measure within the occipital white matter for CEP290-LCA and normal subjects indicate no differences. 
transmit retinal activity to higher visual centers. We conducted a whole-brain, voxel-based morphometric analysis [Ashburner and Friston, 2000] of the anatomical images obtained from Patients P4 and $\mathrm{P} 8$ and found no significant deviations from normal cortical or subcortical anatomy (Fig. 4C). A more focused analysis, conducted within occipital lobe white matter, also did not show a population difference. There were no signs of Joubert syndrome in Patients P4 and P8, confirming previous observations [den Hollander et al., 2006].

\section{DISCUSSION}

CEP290 is a centrosomal protein that also localizes to the cilium of mouse retinal photoreceptors [Chang et al., 2006; Fliegauf et al., 2006; Sayer et al., 2006]. CEP290/NPHP6-associated diseases, including Joubert syndrome and nonsyndromic LCA, now add to the list of human ciliopathies [Badano et al., 2005; Hildebrandt and Otto, 2005; Bisgrove and Yost, 2006; Valente et al., 2006]. The detailed role of CEP290 in the human retina is unknown, except that it causes a profound congenital retinal and visual malfunction [den Hollander et al., 2006; Perrault et al., 2007].

Rod photoreceptor-rich regions of the human CEP290-mutant retina showed a disease phenotype that was comparable to that of the Cep290-deficient $r d 16$ mouse retina. At the earliest human ages studied (7 and 11 years), there was no measurable photoreceptor layer in these rod-rich locations and RPE was demelanized. Assuming allometric scaling of rates of retinal degeneration across species [Wright et al., 2004], the first decade of human life would correspond to about 4 weeks in the life of the rd16 mouse, when severe loss of photoreceptors is already observed [Chang et al., 2006]. Human and murine diseases showed dramatic retinal remodeling in response to the early photoreceptor losses. Abnormal expansion of inner retinal layers was a shared feature. Increased GFAP immunolabeling suggested Muller glial cell activation in response to the genetic photoreceptor injury [Lewis and Fisher, 2003; Rattner and Nathans, 2005]. Muller cell bodies are in the INL and processes project to the outer and inner limiting membranes of the retina [Hogan et al., 1971]. GFAP is expressed at basal levels in Muller glial endfeet (axon terminals) and astrocytes of normal mouse retina [Lewis and Fisher, 2003; Schnitzer, 1988]. Following genetic or environmental insult to the retina, there is progressive Muller cell hypertrophy and upregulation of GFAP synthesis that is detected as large columns throughout the cell [Bringmann and Reichenbach, 2001]. Hypertrophied glial processes may partly explain the retinal thickening, such as in the IPL [Jacobson et al., 2006]. Muller glia also secrete trophic factors for cell survival; diffusion or active transport of such factors to neighboring neurons could lead to increased nuclear activity (such as gene transcription) and consequent thickening of the INL in both CEP290-LCA and rd16 retina [Bok et al., 2002; Zeiss et al., 2006]. Inner retinal abnormalities have been observed in canine and murine retinal degenerations [Strettoi et al., 2002; Fath et al., 2005; Hart et al., 2005; Jones and Marc, 2005; Beltran et al., 2006; Tessitore et al., 2006; Zeiss et al., 2006]. The apparently altered nuclear phenotype with increased nuclear volume in $r d 16$ inner retinal cells has not, to our knowledge, been previously reported. The rd16 mouse may provide a convenient platform to initiate investigations into the basis of this observation and also to determine whether inner retinal dysmorphology can be halted or reversed by therapeutic intervention [Wang et al., 2005].
Primate cone-rich central retina has no equivalent in the roddominated murine retina. Unlike other ciliopathies such as RPGR mutations (Fig. 1) and a Bardet-Biedl syndrome [Azari et al., 2006], the retinas of blind patients who carry CEP290 mutations did not show central degeneration and thinning. Instead, there was relatively normal foveal cone photoreceptor lamination and detectable subjacent RPE, over an age-span of 5 decades. There was no evidence of maldevelopment; the CEP290-mutant fovea had presumably undergone the normally protracted postnatal maturation process of nearly 5 years with increases in cone density due to centripetal movement of cells and cone inner and outer segment differentiation [Yuodelis and Hendrickson, 1986; Mayer et al., 1995; Provis et al., 1998]. Normal INL thickness around the fovea also suggests normal development [Hendrickson, 1994; Provis et al., 2005]. We propose that the spared central island is comprised almost entirely of cone photoreceptor cells, based on the observed thickness data, which are highly reminiscent of human cone density profiles [Curcio et al., 1990] and the decline to subnormal ONL thickness that begins about $1 \mathrm{~mm}$ from the fovea, where the rod:cone ratio starts increasing rapidly [Curcio et al., 1990].

A hypothesis for spared but poorly functioning central cones from birth is that there is defective trafficking through the cilium of key molecular cargo such as phototransduction molecules responsible for visual signaling [Burns and Arshavsky, 2005; Calvert et al., 2006]. The sparing, however, was not complete. Patient P8, the 48-year-old with CEP290 mutations and no light perception, had a more limited spatial extent of intact central retina and RPE than the others; this finding suggests that CEP290-mutant central retinas show slowly progressive degeneration, which may ultimately result in complete loss of foveal cone photoreceptors at later ages. The finding that cell death of cone nuclei is significantly slower than rod photoreceptor loss suggests different roles of CEP290 in these two highly specialized photoreceptor populations or different sensitivities to cell death from a putative intersegmental trafficking defect [Besharse et al., 2003]. Recent data concerning a difference in cone and rod behavior in $r d s / r d s$ mice may also be relevant to the persistence of cone retina in CEP290 mutations. Lack of Rds, which is normally expressed in rods and cones [Molday et al., 1987] resulted in different effects in the two photoreceptor types. Cone cells developed abnormal outer segments and remained viable despite impaired phototransduction efficiency in $r d s / r d s$; in contrast, no rod outer segment development occurred and there was apoptotic death of rods [Farjo et al., 2006].

CEP290-LCA could present an opportunity for visual restoration of the central retina by somatic gene replacement therapy. The more rare RPGRIP1 form of LCA also has some theoretical potential for gene therapy success given the promising results in the murine model of the disease [Pawlyk et al., 2005]. The central retinal preservation in RPGRIP-LCA (Patient P9) [Jacobson et al., 2007b] is interestingly similar to that in CEP290-LCA. RPGRIP1, another photoreceptor ciliary protein, may share pathogenic mechanisms with CEP290 [Chang et al., 2006]. In contrast, the CRB1 and RDH12 forms of LCA have severe retinal disorganization from early human life and are unlikely candidates for somatic gene therapy [Jacobson et al., 2003, 2007a]. Somatic gene therapy in the $r d 16$ mouse, specifically targeting cone photoreceptors, is a key experimental step to take. Further human neuroimaging studies are also warranted, to define the extent of functional reorganization using other sensory modalities [Bavelier and Neville, 2002] and thereby clarify the therapeutic potential in CEP290-LCA. The specter of bilateral amblyopia looms in all early 
visual deprivations [Lewis and Maurer, 2005] and may compromise efficacy from retinal treatment. However, even an improvement in CEP290-LCA from bare light perception to coarse spatial resolution may be a worthy goal for therapy of this otherwise incurable disease.

\section{ACKNOWLEDGMENTS}

We thank Mr. A.J. Roman, Dr. M. Swider, Ms. S. Pearce-Kelling, Ms. M. Doobrajh, Ms. E. Smilko, Dr. C. Murga-Zamalloa, Mr. E. Oh, Ms. L. Jia, Ms. K. Branham, Ms. R. Johnston, and Ms. A. Naidu for their critical help, and Mr. S. Lentz for confocal microscopy. A.S. is a Harold F. Falls Collegiate Professor.

\section{REFERENCES}

Ashburner J, Friston KJ. 2000. Voxel-based morphometry-the methods. Neuroimage 11:805-821.

Avants B, Gee JC. 2004. Geodesic estimation for large deformation anatomical shape averaging and interpolation. Neuroimage 23: S139-S150.

Azari AA, Aleman TS, Cideciyan AV, Schwartz SB, Windsor EA, Sumaroka A, Cheung AY, Steinberg JD, Roman AJ, Stone EM, Sheffield VC, Jacobson SG. 2006. Retinal disease expression in Bardet-Biedl syndrome-1 (BBS1) is a spectrum from maculopathy to retina-wide degeneration. Invest Ophthalmol Vis Sci 47:5004-5010.

Badano JL, Teslovich TM, Katsanis N. 2005. The centrosome in human genetic disease. Nat Rev Genet 6:194-205.

Bavelier D, Neville HJ. 2002. Cross-modal plasticity: where and how? Nat Rev Neurosci 3:443-452.

Beltran WA, Hammond P, Acland GM, Aguirre GD. 2006. A frameshift mutation in RPGR exon ORF15 causes photoreceptor degeneration and inner retina remodeling in a model of X-linked retinitis pigmentosa. Invest Ophthalmol Vis Sci 47:1669-1681.

Besharse JC, Baker SA, Luby-Phelps K, Pazour GJ. 2003. Photoreceptor intersegmental transport and retinal degeneration: a conserved pathway common to motile and sensory cilia. Adv Exp Med Biol 533: 157-164.

Bisgrove BW, Yost HJ. 2006. The roles of cilia in developmental disorders and disease. Development 133:4131-4143.

Bok D, Yasumura D, Matthes MT, Ruiz A, Duncan JL, Chappelow AV, Zolutukhin S, Hauswirth W, LaVail MM. 2002. Effects of adenoassociated virus-vectored ciliary neurotrophic factor on retinal structure and function in mice with a P216L rds/peripherin mutation. Exp Eye Res 74:719-735.

Breuer DK, Yashar BM, Filippova E, Hiriyanna S, Lyons RH, Mears AJ, Asaye B, Acar C, Vervoort R, Wright AF, Musarella MA, Wheeler P, MacDonald I, Iannaccone A, Birch D, Hoffman DR, Fishman GA, Heckenlively JR, Jacobson SG, Sieving PA, Swaroop A. 2002. A comprehensive mutation analysis of RP2 and RPGR in a North American cohort of families with X-linked retinitis pigmentosa. Am J Hum Genet 70:1545-1554.

Bringmann S, Reichenbach A. 2001. Role of Muller cells in retinal degenerations. Front Biosci 6:e77-e92.

Burns ME, Arshavsky VY. 2005. Beyond counting photons: trials and trends in vertebrate visual transduction. Neuron 48:387-401.

Calvert PD, Strissel KJ, Schiesser WE, Pugh EN Jr, Arshavsky VY. 2006. Light-driven translocation of signaling proteins in vertebrate photoreceptors. Trends Cell Biol 16:560-568.

Chang B, Khanna H, Hawes N, Jimeno D, He S, Lillo C, Parapuram SK, Cheng H, Scott A, Hurd RE, Sayer JA, Otto EA, Attanasio M, O’Toole JF, Jin G, Shou C, Hildebrandt F, Williams DS, Heckenlively JR, Swaroop A. 2006. In-frame deletion in a novel centrosomal/ciliary protein CEP290/NPHP6 perturbs its interaction with RPGR and results in earlyonset retinal degeneration in the rd16 mouse. Hum Mol Genet 15: $1847-1857$.

Cideciyan AV, Swider M, Aleman TS, Roman MI, Sumaroka A, Schwartz SB, Stone EM, Jacobson SG. 2007. Reduced illuminance autofluores- cence imaging in ABCA4-associated retinal degenerations. J Opt Soc Am A 24:1457-1467.

Cremers FP, van den Hurk JA, den Hollander AI. 2002. Molecular genetics of Leber congenital amaurosis. Hum Mol Genet 11:1169-1176.

Curcio CA, Sloan KR, Kalina RE, Hendrickson AE. 1990. Human photoreceptor topography. J Comp Neurol 292:497-523.

den Hollander AI, Koenekoop RK, Yzer S, Lopez I, Arends ML, Voesenek KE, Zonneveld MN, Strom TM, Meitinger T, Brunner HG, Hoyng CB, van den Born LI, Rohrschneider K, Cremers FP. 2006. Mutations in the CEP290 (NPHP6) gene are a frequent cause of Leber congenital amaurosis. Am J Hum Genet 79:556-561.

Eley L, Yates LM, Goodship JA. 2005. Cilia and disease. Curr Opin Genet Dev 15:308-314.

Farjo R, Skaggs JS, Nagel BA, Quiambao AB, Nash ZA, Fliesler SJ, Naash MI. 2006. Retention of function without normal disc morphogenesis occurs in cone but not rod photoreceptors. J Cell Biol 173:59-68.

Fath MA, Mullins RF, Searby C, Nishimura DY, Wei J, Rahmouni K, Davis RE, Tayeh MK, Andrews M, Yang B, Sigmund CD, Stone EM, Sheffield VC. 2005. Mkks-null mice have a phenotype resembling Bardet-Biedl syndrome. Hum Mol Genet 14:1109-1118.

Fliegauf M, Horvath J, von Schnakenburg C, Olbrich H, Muller D, Thumfart J, Schermer B, Pazour GJ, Neumann HP, Zentgraf H, Benzing T, Omran H. 2006. Nephrocystin specifically localizes to the transition zone of renal and respiratory cilia and photoreceptor connecting cilia. J Am Soc Nephrol 17:2424-2433.

Hart AW, McKie L, Morgan JE, Gautier P, West K, Jackson IJ, Cross SH. 2005. Genotype-phenotype correlation of mouse pde6b mutations. Invest Ophthalmol Vis Sci 46:3443-3450.

Hendrickson AE. 1994. Primate foveal development: a microcosm of current questions in neurobiology. Invest Ophthalmol Vis Sci 35: 3129-3133.

Hildebrandt F, Otto E. 2005. Cilia and centrosomes: a unifying pathogenic concept for cystic kidney disease? Nat Rev Genet 6:928-940.

Hogan MJ, Alvarado JA, Weddell JE. 1971. Histology of the human eye. Philadelphia: WB Saunders Company. p 393-522.

Huang Y, Cideciyan AV, Papastergiou GI, Banin E, Semple-Rowland SL, Milam AH, Jacobson SG. 1998. Relation of optical coherence tomography to microanatomy in normal and rd chickens. Invest Ophthalmol Vis Sci 39:2405-2416.

Huang Y, Cideciyan AV, Aleman TS, Banin E, Huang J, Syed NA, Petters RM, Wong F, Milam AH, Jacobson SG. 2000. Optical coherence tomography (OCT) abnormalities in rhodopsin mutant transgenic swine with retinal degeneration. Exp Eye Res 70:247-251.

Jacobson SG, Voigt WJ, Parel JM, Apathy PP, Nghiem-Phu L, Myers SW, Patella VM. 1986. Automated light- and dark-adapted perimetry for evaluating retinitis pigmentosa. Ophthalmology 93:1604-1611.

Jacobson SG, Cideciyan AV, Aleman TS, Pianta MJ, Sumaroka A, Schwartz SB, Smilko EE, Milam AH, Sheffield VC, Stone EM. 2003. Crumbs homolog 1 (CRB1) mutations result in a thick human retina with abnormal lamination. Hum Mol Genet 12:1073-1078.

Jacobson SG, Aleman TS, Cideciyan AV, Sumaroka A, Schwartz SB, Windsor EA, Traboulsi EI, Heon E, Pittler SJ, Milam AH, Maguire AM, Palczewski K, Stone EM, Bennett J. 2005. Identifying photoreceptors in blind eyes caused by RPE65 mutations: prerequisite for human gene therapy success. Proc Natl Acad Sci USA 102: 6177-6182.

Jacobson SG, Cideciyan AV, Sumaroka A, Aleman TS, Schwartz SB, Windsor EA, Roman AJ, Stone EM, MacDonald IM. 2006. Remodeling of the human retina in choroideremia: rab escort protein 1 (REP-1) mutations. Invest Ophthalmol Vis Sci 47:4113-4120.

Jacobson SG, Cideciyan AV, Aleman TS, Sumaroka A, Schwartz SB, Windsor EAM, Roman AJ, Heon E, Stone EM, Thompson DA. 2007a. RDH12 and RPE65, visual cycle genes causing Leber congenital amaurosis, differ in disease expression. Invest Ophthalmol Vis Sci 48: 332-338.

Jacobson SG, Cideciyan AV, Aleman TS, Sumaroka A, Schwartz SB, Roman AJ, Stone EM. 2007b. Leber congenital amaurosis caused by RPGRIP1 mutation shows treatment potential. Ophthalmolgy 114: 895-898. 
Jones BW, Marc RE. 2005. Retinal remodeling during retinal degeneration. Exp Eye Res 81:123-137.

Karim S, Clark RA, Poukens V, Demer JL. 2004. Demonstration of systematic variation in human intraorbital optic nerve size by quantitative magnetic resonance imaging and histology. Invest Ophthalmol Vis Sci 45:1047-1051.

Keilhauer CN, Delori FC. 2006. Near-infrared autofluorescence imaging of the fundus: visualization of ocular melanin. Invest Ophthalmol Vis Sci 47:3556-3564.

Lewis GP, Fisher SK. 2003. Up-regulation of glial fibrillary acidic protein in response to retinal injury: its potential role in glial remodeling and a comparison to vimentin expression. Int Rev Cytol 230:263-290.

Lewis TL, Maurer D. 2005. Multiple sensitive periods in human visual development: evidence from visually deprived children. Dev Psychobiol 46:163-183.

Lotery AJ, Jacobson SG, Fishman GA, Weleber RG, Fulton AB, Namperumalsamy P, Heon E, Levin AV, Grover S, Rosenow JR, Kopp KK, Sheffield VC, Stone EM. 2001. Mutations in the CRB1 gene cause Leber congenital amaurosis. Arch Ophthalmol 119:415-420.

Mayer DL, Beiser AS, Warner AF, Pratt EM, Raye KN, Lang JM. 1995. Monocular acuity norms for the Teller acuity cards between ages one month and four years. Invest Ophthalmol Vis Sci 36:671-685.

Molday RS, Hicks D, Molday L. 1987. Peripherin. A rim-specific membrane protein of rod outer segment discs. Invest Ophthalmol Vis Sci 28:50-61.

Noppeney U, Friston KJ, Ashburner J, Frackowiak R, Price CJ. 2005. Early visual deprivation induces structural plasticity in gray and white matter. Curr Biol 15:R488-R490.

Pan J, Wang Q, Snell WJ. 2005. Cilium-generated signaling and ciliarelated disorders. Lab Invest 85:452-463.

Parisi MA, Doherty D, Chance PF, Glass IA. 2007. Joubert syndrome (and related disorders) (OMIM 213300). Eur J Hum Genet 15: 511-521.

Pawlyk BS, Smith AJ, Buch PK, Adamian M, Hong DH, Sandberg MA, Ali RR, Li T. 2005. Gene replacement therapy rescues photoreceptor degeneration in a murine model of Leber congenital amaurosis lacking RPGRIP. Invest Ophthalmol Vis Sci 46:3039-3045.

Perrault I, Delphin N, Hanein S, Gerber S, Dufier J-L, Roche O, DefoortDhellemmes S, Dollfus H, Fazzi E, Munnich A, Kaplan J, Rozet J-M. 2007. Spectrum of NPHP6/CEP290 mutations in Leber congenital amaurosis and delineation of the associated phenotype. Hum Mutation 28:416.

Provis JM, Diaz CM, Dreher B. 1998. Ontogeny of the primate fovea: a central issue in retinal development. Prog Neurobiol 54:549-580.

Provis JM, Penfold PL, Cornish EE, Sandercoe TM, Madigan MC. 2005. Anatomy and development of the macula: specialisation and the vulnerability to macular degeneration. Clin Exp Optom 88:269-281.
Rattner A, Nathans J. 2005. The genomic response to retinal disease and injury: evidence for endothelin signaling from photoreceptors to glia. J Neurosci 25:4540-4549.

Saunier S, Salomon R, Antignac C. 2005. Nephronophthisis. Curr Opin Genet Dev 15:324-331.

Sayer JA, Otto EA, O'Toole JF, Nurnberg G, Kennedy MA, Becker C, Hennies HC, Helou J, Attanasio M, Fausett BV, Utsch B, Khanna H, Liu Y, Drummond I, Kawakami I, Kusakabe T, Tsuda M, Ma L, Lee H, Larson RG, Allen SJ, Wilkinson CJ, Nigg EA, Shou C, Lillo C, Williams DS, Hoppe B, Kemper MJ, Neuhaus T, Parisi MA, Glass IA, Petry M, Kispert A, Gloy J, Ganner A, Walz G, Zhu X, Goldman D, Nurnberg P, Swaroop A, Leroux MR, Hildebrandt F. 2006. The centrosomal protein nephrocystin- 6 is mutated in Joubert syndrome and activates transcription factor ATF4. Nat Genet 38:674-681.

Schnitzer J. 1988. Immunocytochemical studies on the development of astrocytes, Muller (glial) cells, and oligodendrocytes in the rabbit retina. Brain Res Dev Brain Res 44:59-72.

Shimony JS, Burton H, Epstein AA, McLaren DG, Sun SW, Snyder AZ. 2006. Diffusion tensor imaging reveals white matter reorganization in early blind humans. Cereb Cortex 16:1653-1661.

Strettoi E, Porciatti V, Falsini B, Pignatelli V, Rossi C. 2002. Morphological and functional abnormalities in the inner retina of the $\mathrm{rd} / \mathrm{rd}$ mouse. J Neurosci 22:5492-5504.

Tessitore A, Parisi F, Denti MA, Allocca M, Di Vicino U, Domenici L, Bozzoni I, Auricchio A. 2006. Preferential silencing of a common dominant rhodopsin mutation does not inhibit retinal degeneration in a transgenic model. Mol Ther 14:692-699.

Valente EM, Silhavy JL, Brancati F, Barrano G, Krishnaswami SR, Castori M, Lancaster MA, Boltshauser E, Boccone L, Al-Gazali L, Fazzi E, Signorini S, Louie CM, Bellacchio E, International Joubert Syndrome Related Disorders Study Group, Bertini E, Dallapiccola B, Gleeson JG. 2006. Mutations in CEP290, which encodes a centrosomal protein, cause pleiotropic forms of Joubert syndrome. Nat Genet 38: 623-625.

Wang S, Lu B, Lund RD. 2005. Morphological changes in the Royal College of Surgeons rat retina during photoreceptor degeneration and after cell-based therapy. J Comp Neurol 491:400-417.

Wright AF, Jacobson SG, Cideciyan AV, Roman AJ, Shu X, Vlachantoni D, McInnes RR, Riemersma RA. 2004. Lifespan and mitochondrial control of neurodegeneration. Nat Genet 36:1153-1158.

Wright AF, Shu X. Focus on molecules: RPGR. Exp Eye Res (in press).

Yuodelis C, Hendrickson A. 1986. A qualitative and quantitative analysis of the human fovea during development. Vision Res 26:847-855.

Zeiss CJ, Allore HG, Towle V, Tao W. 2006. CNTF induces dose-dependent alterations in retinal morphology in normal and rcd-1 canine retina. Exp Eye Res 82:395-404. 